\title{
EVALUATION OF RISK FACTORS FOR BREAST CANCER IN PAKISTANI WOMEN
}

\author{
Zeba Hisam ${ }^{1}$ Noor Muhammad Soomro ${ }^{2}$, Rasheed Ahmed Lakhiar ${ }^{3}$
}

\author{
'Assistant Professor \& Head of Department Physiology, Fatima Jinnah Dental \\ College \\ ${ }^{2}$ Chief Oncologist \& HOD Oncology Unit, Civil Hospital Kartachi \\ ${ }^{3}$ Senior Medical Officer, Oncology Unit, Civil Hospital Karachi \\ Corresponding Author:Zeba Hisam,Email: zhisam@pakdata.com
}

\begin{abstract}
Background: Breast cancer is one of the leading causes of morbidity and mortility in females of pakistan. We conducted this study to evaluate risk factors for developing breast cancer in Pakistani women.

Methods: Study Design: An observational/ cross sectional study was conducted at Dr. Ruth Pfau K. M Civil Hospital Karachi, Oncology Ward, from May 2017 to February 2018. A total of 80 patients of biopsy positive cases of breast cancer in Pakistani females were included who were admitted for chemotherapy. By taking the histories, their risk factors were evaluated, weight, height recorded and BMI calculated.

Results: In this study 80 females were included to evaluate the risk factors for cancer of breast. Age was $40-59$ years in $46.3 \%$ and $32.5 \%$ were of 24-39 year. Out of total, 98.8\% were married, 1.3\% was unmarried. Family history of breast cancer was $17.5 \%$, early menarche $7.5 \%$, late menopause $11.8 \%$. Nulliparity was $10 \%$ and multiparity was $90 \%$. Age at first full-term pregnancy above 35 years was $1.3 \%$. Breast-feeding was in $77.5 \%$ and oral contraceptive pills use $18.8 \%$. HRT was absent $100 \%$. Dietary intake of high fat $40 \%$, lack of exercise $95 \%$, raised BMI $78.8 \%$ and use of hair dye in $58.8 \%$. Synthetic dye use was reported in $4 \%$ and Henna was used in $96 \%$. Frequency of hair dye use, ranged from occasional (4-5 times/year) $4.2 \%$, to twice a month $4.2 \%$ and monthly $91.6 \%$. Duration of hair dye use reported was for 2 years $(4 \%), 5$ years $(7 \%), 7$ years $(11 \%), 10$ years $(14 \%), 15$ years $(20 \%)$ and 20 years $(28 \%)$.

Conclusion: The risk factors for cancer of breast have a different implication on Pakistani women than their western counterparts. Genetic and hormonal factors play a smaller role while life style factors play a major role including a recently associated risk factor of the use of hair dye.
\end{abstract}

Keywords: Breast cancer, risk factors, hair dye. 


\section{Introduction}

The most common cancer among women is the breast cancer. Globally, about 1.38 million women suffer from cancer of breast and about 458,000 die of this malignancy each year. The incidence and mortality of breast cancer has increased in Asian and African countries due to poor health facilities and absence of screening mammography(1). Pakistan is a developing country and WHO classifies it as of low to medium category. According to the GLOBOCAN, which is a project of the International Agency for Research on Cancer (IARC) reveals estimates by cancer site and sex by using the best available data in each country and by many methods of estimation. (2) GLOBOCAN data of 2018 reveals that most common cancer among females is the breast cancer in majority of countries (154 0f 185). Incidence rates of breast cancer are highest in Australia/New Zealand, Northern Europe (United Kingdom, Sweden, Finland and Denmark), Western Europe (Belgium with highest global rates), Netherlands and France. In the United States lung cancer incidence has surpassed breast cancer. Breast cancer incidence rates are rising in transitioning countries in Asia and Africa. (3) GLOBOCAN data of 2012 revealed that most common malignancy for women in Pakistan is the malignancy of breast $(n=34038,23 \%)$. (4).

In Pakistan, incidence of breast cancer is rising with alarming rates The Karachi Cancer Registry (KCR) data (1998-2002) shows a rise of malignancies during the last decade, more significantly in women. A surge in breast cancer incidence is seen among females with maximum records rates for younger aged women. The Aga Khan University Surveillance for Pakistan (ASCP) with the help of KCR has estimated for Karachi division (1998-2002), the highest incidence for breast malignancy among females (53.1). The Incidence of breast cancer is maximum in Karachi; the highest in Asia, except for the Jews in Israel. (5) Younger women are being diagnosed with breast malignancy. Due to lack of awareness, education, poverty and social taboos related to female body, many women do not approach a doctor despite finding a lump or other changes in the breast as they cannot think of exposing their breast to a stranger, even a female doctor. Instead, many women go to spiritual healers and quacks, mostly male, who treat them by listening to their symptoms, misguide them and ultimately cancer is advanced. The most alarming signs, which force them to seek medical advice, are bursting of breast lump discharging pus and blood leading to non-healing breast wounds.

It has been observed that in many women in Pakistan who have breast cancer, do not have the known risk factors. Hence, a factor that has been recently linked to the incidence of developing breast cancer is included in the present study, that is the use of hair dye. (6)

One of the crucial established risk factor for the occurrence of breast cancer is age. The risk increases with increasing age. In western countries, the incidence of breast cancer increases until 60s, peaks in $70 \mathrm{~s}$, then decreases. Family history of breast cancer is another important risk for development of breast cancer including genetic mutation of BRCA1 (present on chromosome 17) and BRCA2 (present on chromosome 13) or tumour suppressor gene P53. (7)

Other established risk factors such as early menarche (before age 12), late menopause (after age 50), nulliparity and age at first full-term pregnancy above 35 years, impose a greater risk for breast cancer. Use of oral contraceptive pills increases the incidence. (8) Use of hormone replacement therapy by postmenopausal females may significantly raise the risk of breast cancer. This link is confirmed by many other studies including the Million Women Study. (9) Certain life style factors are also linked with the incidence of breast cancer. Alcohol consumption, increased intake of fatty diet and sedentary lifestyle may also raise the incidence. Obesity also plays a crucial role and raises the risk of developing cancer of breast by increasing the levels of oestrogen in the body. (10) Proliferative changes in the breast also increase the incidence of cancer of breast. (11-12) Atypical hyperplasia of epithelium is also linked with increased incidence. Any benign proliferative change in the mammary gland can be transformed into malignant lesion later. (13) Hair dye use is also being linked with increased incidence of breast cancer development. There are two types of modern hair dyes. First type is oxidative dye which includes colourless intermediate (Aromatic amines) and dye couplers. In the presence of hydrogen peroxide, the intermediates and couplers react to produce molecules of pigment which stain the hair permanently. The other type of hair dye is non oxidative (temporary or semi permanent) which contains the coloured chemicals which stain the hairs directly.
Several chemicals are used in hair dyes (about 5000) and some of them are mutagenic. (14)

\section{Methodology}

The data for this observational/cross sectional study was collected over the period of ten months (May 2017 to Feb 2018) from the Oncology ward of Dr. Ruth Pfau K. M Civil Hospital Karachi. Inclusion criteria were histologically confirmed cases of breast cancer that were admitted for chemotherapy. In this study, 80 patients were included. Histories were taken to assess the risk factors. Weight and height were recorded and BMI calculated. These patients were referred to the Civil Hospital from different neighbourhoods of Karachi and from other cities of the province of Sindh including Mirpurkhas, Larkana, Naushero Feroz, Nawabshah, Dadu, Thatta, Sukkar, Moro, Khairpur, Shikarpur, Hyderabad and Ghotki. From the Punjab province, the patients came from Mianwali, Rahim Yar Khan and Rajanpur. From Baluchistan province, patients' residence was reported to be Khuzdar, Jacobabad, Turbat and Gwadar.

Descriptive statistics were obtained for the risk factors including age, marital status, breast feeding, menarche age, first full-term pregnancy age, menopausal age, family history of cancer of breast, previous history of benign fibrocystic disease or cancer of other breast, use of oral contraceptive pills, therapy for hormone replacement, dietary habits, exercise and the BMI and use of hair dye. Analysis of data was done on Statistical Package of Social Sciences (SPSS).

\section{Results}

Out of 80 patients, twenty-six women were $24-39$ years of age $(32.5 \%)$; 37 women were $40-59$ years of age $(46.3 \%)$ and 17 women were above 60 years $(17 \%)$ (Table 1$)$. Out of all, 79 were married $(98.8 \%)$ and one was unmarried $(1.3 \%)$. History of cancer of breast in the family was positive in 14 cases $(17.5 \%)$ and negative in 66 cases $(82.5 \%)$. Previous medical history of benign fibrocystic disease or cancer in other breast was present in 11 cases $(13.8 \%)$ and negative in 69 cases $(86.3 \%)$.

Early menarche was in 6 cases $(7.5 \%)$ and negative in 74 cases $(92.5 \%)$. Late menopause was found in 9 cases $(11.3 \%)$ and negative in 71 cases $(88.8 \%)$. Nulliparous women were $8(10 \%)$ and multiparous were $72(90 \%)$. First full term pregnancy above 35 years was found in only 1 case $(1.3 \%)$ and negative in 79 cases $(98.8 \%)$. Use of oral contraceptive pills was in 15 cases $(18.8 \%)$ and negative in 65 cases $(81.3 \%)$. HRT was negative in all $(100 \%)$. Breast-feeding was positive in 62 cases $(77.5 \%)$ and negative in 18 cases $(22.5 \%)$. Intake of high fatty diet was in 32 cases $(40 \%)$ and negative in 48 cases $(60 \%)$. Exercise was a habit in only 4 cases $(5.0 \%)$ and lack of exercise was present in 76 cases $(95 \%)$. BMI was between 18.5-24.9 in 7 cases $(8.8 \%), 25-30$ in 63 cases $(78.8 \%)$ and 31 and above was in 10 cases $(12.5 \%)$. Use of hair dye was present in 47 women $(58.8 \%)$ and negative in $33(41.3 \%)$ (figure 2$)$. Out of these, 45 used natural herb Henna (95.7\%) and 2 used synthetic dye (4.3\%) (figure 3). Frequency of hair dye use, ranged from occasional (4-5 times/year) $4.2 \%$, Twice a month $4.2 \%$ and monthly $91.6 \%$ (Figure 4 ). Duration of hair dye use reported was for 2 years $(4 \%), 5$ years $(7 \%), 7$ years $(11 \%), 10$ years $(14 \%), 15$ years $(20 \%)$ and 20 years $(28 \%)$ (Figure 5$)$.

As the natural herb "Henna" that is commonly available in Pakistan, is mixed with many unknown chemicals so contamination is possible. 
Table 1 Percentages of Risk Factors for Developing Cancer of Breast in Pakistani Females

\begin{tabular}{|c|c|c|c|c|}
\hline Age & Frequency & Per cent & Valid Per cent & Cumulative Per cent \\
\hline $24-39$ & 26 & 32.5 & 32.5 & 32.5 \\
\hline $40-59$ & 37 & 46.3 & 46.3 & 78.8 \\
\hline 60 and above & 17 & 21.3 & 21.3 & 100 \\
\hline Total & 80 & 100 & 100 & \\
\hline \multicolumn{5}{|l|}{ Marital Status } \\
\hline Married & 79 & 98.8 & 98.8 & 98.8 \\
\hline Unmarried & 1 & 1.3 & 1.3 & 100 \\
\hline Total & 80 & 100 & 100 & \\
\hline \multicolumn{5}{|c|}{ Family History of Breast Cancer } \\
\hline Yes & 14 & 17.5 & 17.5 & 17.5 \\
\hline No & 66 & 82.5 & 82.5 & 100 \\
\hline Total & 80 & 100 & 100 & \\
\hline \multicolumn{5}{|c|}{$\begin{array}{l}\text { Previous medical history (Fibrocystic } \\
\text { disease or cancer in other breast }\end{array}$} \\
\hline Yes & 11 & 13.8 & 13.8 & 13.8 \\
\hline No & 69 & 86.3 & 86.3 & 100 \\
\hline Total & 80 & 100 & 100 & \\
\hline \multicolumn{5}{|c|}{ Early menarche (under age 12) } \\
\hline Yes & 6 & 7.5 & 7.5 & 7.5 \\
\hline No & 74 & 92.5 & 92.5 & 100 \\
\hline Total & 80 & 100 & 100 & \\
\hline \multicolumn{5}{|c|}{ Late menopause (Above age 50) } \\
\hline Yes & 9 & 11.3 & 11.3 & 11.3 \\
\hline No & 71 & 88.8 & 88.8 & 100 \\
\hline Total & 80 & 100 & 100 & \\
\hline \multicolumn{5}{|c|}{ Reproductive history } \\
\hline Nulliparous & 8 & 10 & 10 & 10 \\
\hline Multiparous & 72 & 90 & 90 & 100 \\
\hline Total & 80 & 100 & 100 & \\
\hline \multicolumn{5}{|c|}{ 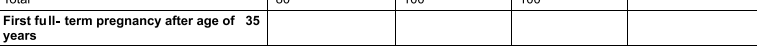 } \\
\hline Yes & 1 & 1.3 & 1.3 & 1.3 \\
\hline No & 79 & 98.8 & 98.8 & 100 \\
\hline \multirow[t]{2}{*}{ Total } & 80 & 100 & 100 & \\
\hline & Frequency & Per cent & Valid Per cent & Cumulative Per cent \\
\hline \multicolumn{5}{|c|}{ Use of oral contraceptive pills } \\
\hline Yes & 15 & 18.8 & 18.8 & 18.8 \\
\hline No & 65 & 81.3 & 81.3 & 100 \\
\hline Total & 80 & 100 & 100 & \\
\hline \multicolumn{5}{|c|}{ 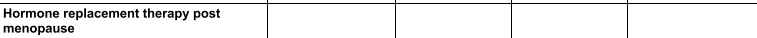 } \\
\hline No & 80 & 100 & 100 & 100 \\
\hline Total & 80 & 100 & 100 & \\
\hline \multicolumn{5}{|c|}{ Breast Feeding } \\
\hline Yes & 62 & 77.5 & 77.5 & 77.5 \\
\hline No & 18 & 22.5 & 22.5 & 100 \\
\hline Total & 80 & 100 & 100 & \\
\hline \multicolumn{5}{|c|}{ Diet (Fatty food) } \\
\hline Yes & 32 & 40 & 40 & 40 \\
\hline No & 48 & 60 & 60 & 100 \\
\hline Total & 80 & 100 & 100 & \\
\hline \multicolumn{5}{|l|}{ EXCERSICE } \\
\hline Yes & 4 & 5 & 5 & 5 \\
\hline No & 76 & 95 & 95 & 100 \\
\hline Total & 80 & 100 & 100 & \\
\hline \multicolumn{5}{|l|}{ BMI } \\
\hline $18.5-24.9$ & 7 & 8.8 & 8.8 & 8.8 \\
\hline $25-30$ & 63 & 78.8 & 78.8 & 87.5 \\
\hline 31 and above & 10 & 12.5 & 12.5 & 100 \\
\hline Total & 80 & 100 & 100 & \\
\hline Use of hair dye & & & & \\
\hline Yes & 47 & 58.8 & 58.8 & 58.8 \\
\hline No & 33 & 41.3 & 41.3 & 100 \\
\hline Total & 80 & 100 & 100 & \\
\hline Type of hair dy & & & & \\
\hline Henna & 45 & 95.7 & 95.7 & 95.7 \\
\hline Synthetic dye & 2 & 4.3 & 4.3 & 100 \\
\hline Total & 47 & 100 & 100 & \\
\hline
\end{tabular}

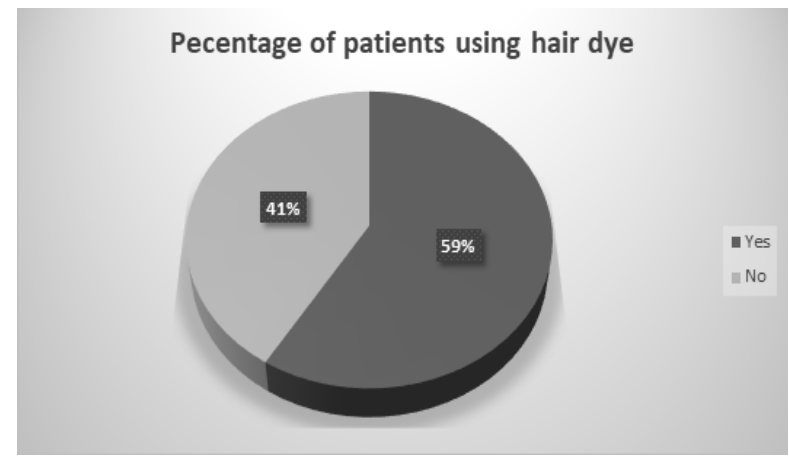

Figure 2: Pie chart showing the distribution of patients using hair dye

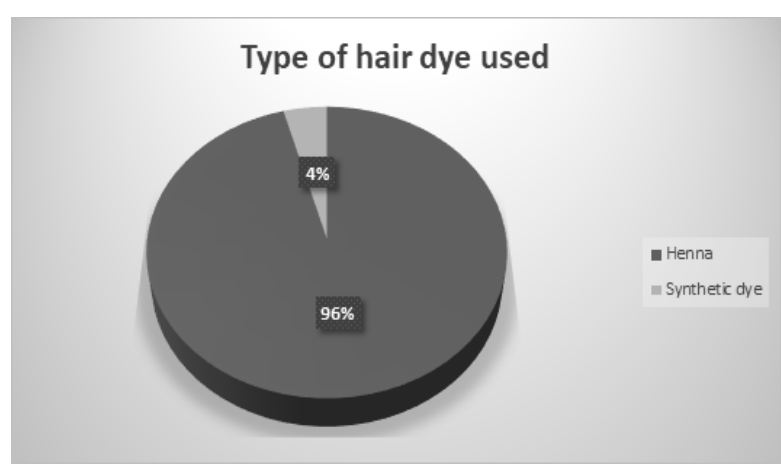

Figure 3: Pie chart showing types of hair dye used

Frequancy of hair dye use

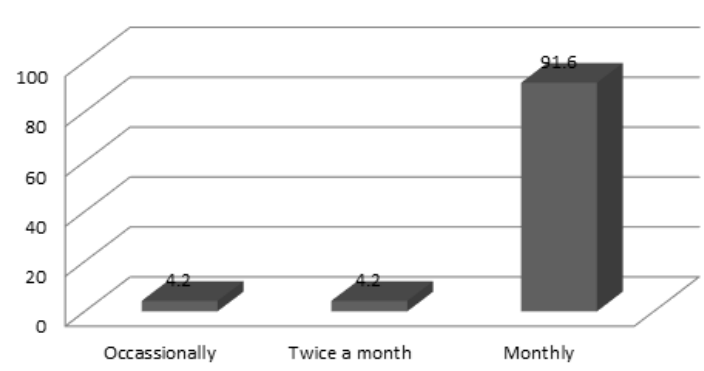

Figure 4: Pie chart showing frequency of hair dye use

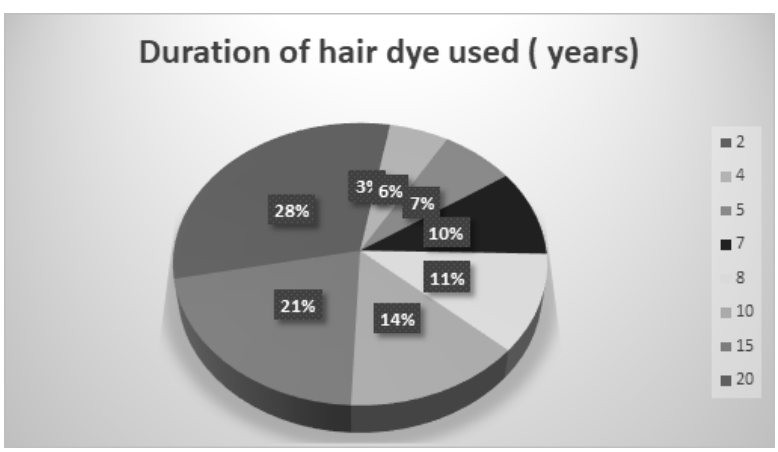

Figure 5: Pie chart showing duration of hair dye used in years

\section{Discussion}

In the present study we assessed the risk factors linked with the increased incidence of developing cancer of breast in Pakistani females. The risk of cancer of breast is increasing in Pakistan and younger females are also being diagnosed with the disease. Breast cancer is a hormone dependent disease so age at menarche, first fullterm pregnancy age and menopausal age have a greater implication on the development of cancer of breast. (15) Environmental and life style factors also play a role in its incidence. In present study we found out that most women who were diagnosed with breast cancer were of ages between $40-59$ years $(46.3 \%)$. A study carried out at Shaukat Khanum Memorial Hospital, Lahore, Pakistan, Farhana Badar et al, reported that mean age of breast cancer is 48.6 years in Pakistani women. Another study carried out at Nawabshah, Gulshan Ali Memon et al, reported the common age group for developing breast cancer was found to be 44-48 years. (16) In one similar study, it was reported that most of the cancers of breast developed in females at 35-45 years. (17) In the present study, younger women were also found to have breast cancer at age 24-39 years (32.5\%). Another study conducted in Lahore, Mansha $\mathrm{M}$ et al, also reported that most of the cancers of breast occurred in Pakistani females between 35-45 years age. (18)

T. Pervez et al conducted a similar study to establish the increased incidence of cancer of breast in population in Lahore. According to 
that, history of breast cancer in family, oral contraceptive pills use, early menarche and infertility were found to be significant risk factors whereas, positive breast-feeding and late childbearing had no significant implication on development of cancer of breast in Pakistani females. In present study, breast cancer development was found with positive family history of breast cancer in $17.5 \%$ cases. (19) In the present study we also found that breast cancer developed in most women despite having protective factors against breast cancer, like menarche after 12 years $(92.5 \%)$, menopause before 50 years $(88.8 \%)$, age at first full term pregnancy less than 35 years ( $98.8 \%$ ), nulliparity in only $10 \%$ and positive breast-feeding $(77.5 \%)$. HRT was absent in all $100 \%$ in this study.

Similar results were found in a study carried out in Lahore, showed that factors for the increased incidence of breast cancer like infertility, old age, menopause after the age of 50 and history of breast cancer in a family bear a different connection to the development of cancer of breast in Pakistani females. (20) Another study conducted in Islamabad found that absence of breast-feeding; nulliparity, late menopause and oral contraceptive pills use increase the incidence of cancer of breast. (23).

In the present study, use of oral contraceptive pills was $18.8 \%$. According to a study, Lina S. Morch, et al reported that oral contraceptive pills use increases the risk for development of cancer of breast in women. (2 Environmental factors showed more contribution for incidence of bre)ast cancer. A study by Memon Z. A et al, reveals that high fat diet increases the incidence of cancer of breast. In this study, dietary habits showed that women consumed high fatty diet $(40 \%)$. Lack of exercise was present in the majority of women $(95 \%)$ and BMI was elevated 25-30 in (78.8\%). Another study carried out in Karachi, Rukhsana Khursheed, et al revealed the same results that lack of exercise, sedentary life and increased intake of fatty diet poses a risk for breast cancer. (24)

Some latest studies show that use of hair dye may also increase the incidence of cancer of breast as exposure to carcinogen in hair products have been explored. According to one study, Llanos AAM et al reviewed 4300 women. They found that dark hair dyes applications and higher frequency of use was linked with increased incidence of cancer of breast. These findings establish a connection between the hair products applications and development of cancer of breast. Although a statistical association is observed between application of hair dye and development of breast cancer but cause-effect relationship has not yet been established. This study established a correlation and not a causative link. Another retrospective population-based casecontrol study in Finland showed that the link between application of hair dye and occurrence of cancer of breast was present among females aged 22-60 years. In the present study, use of hair dye was also assessed and most women were found to use hair dye $(58.8 \%)$. Although all women belonged to low socioeconomic status and most of them used 'henna' to dye their hair, two of them used synthetic dyes also. Natural herb henna is supposed to be a safe natural dye for colouring the hair. But henna available in Pakistan, is commonly being mixed with unknown chemicals which could be harmful and mutagenic. Our study has also found an association between the application of hair dye and occurrence of cancer of breast.

\section{Conclusion}

Age, history of breast cancer in family, menarche at an early age, late first full-term pregnancy, menopause at older age, use of oral contraceptive pills and HRT have smaller contribution in the development of cancer of breast in Pakistani females. Life style factors like high fate intake, lack of exercise and raised BMI seem contribute more in the development of cancer of breast. (23) A non-established risk factor of hair dye use, suggests that hair dye exposure can be linked with increased incidence of cancer of breast in our study. This was a small study so larger group of patients need to be studied for the risk factors for breast cancer development.

References

1.

Anderson KN et al. Current Medical Diagnosis \& Treatment. 2017 ed.Mc-Graw-Hill education, 2017: 737738

2. Sebastien Antoni, Isabelle Soerjomataram, Bjorn Moller, Freddie Bray \& Jacques Ferlay. An assessment of GLOBOCAN methods for deriving national estimates of cancer incidence. Bulletin of the World Health Organization 2016;94: 174-184
3. Freddy Bray, Jacques Ferlay, Isabella Soerjomataram, Rebecca L. Siegel, Lindsay A. Torre, et.al of incidence and mortality wo. Global Cancer Statistics 2018: GLOBOCAN estimates of Incidence and Mortality Worldwide for 36 Cancers in 185 Countries.CA2018: Vol (68); 6

4. Muhammad Rehan Sarwar, Anum Saqib. Mortality rates in Pakistan. Cogent medicine 4 (1), 1288773, 2017

5. Yasmin Bhrgri, Asif Bhurgri, Sania Nishter, Ashfaq Ahmed, Ahmed Usman. Pakistan-Country Profile of Cancer and Cancer Control 1995-2002. JPMA, March 2006 Lanos AAM, Rabkin A, Bandersa EV, Zirpoli G, Gozalez $\mathrm{BD}$, Xing Cy. et al. Hair product use and breast cancer risk among African American and white women. J Carcinog 2017; Sep 1:38(9): 883-892

7. Tariq Moatter, Muniba Aban, Shaista khan, Iqbal Azam and Shahid Pervez. BRCA1 Status in Pakistani Breast Cancer Patients with Moderate Family History. JCPSP 2011; Vol. 21 (11): 680-684

8. Chris Kahlenborn, Walter B. Severs. Oral Contraceptive Use as a Risk Factor for Premenopausal Breast Cancer: A meta-analysis. Science Direct. Oct 2006; 81: 2090-1302

9. Narod SA. Hormone Replacement therapy and Risk of Breast Cancer. Nat Rev Clin Oncol. 2011

10. K. McPherson, C M. Steel, and J M. Dixon. Breast Cancer Epidemiology, risk factors, and genetics. BMJ 2000; 11: 321 97270: 1198

11. Lynn C. Hartmann, MD., Amy C. Degnim M.D., Richard J. Santen M.D, William D. Dupont, Ph. D., et al. Atypical Hyperplasia of the Breast- Risk Assessment and Management Options. N Engl j Med 2015: 372:78-89

12. Aisha Memon, Shahida Parveen, A. K Sangrarasi et al. Changing Patterns of Benign Breast Lumps in Young Females World j. Med. Sci. 2007; 2 (1); 21-24

13. Menopauzalny, 2015 Sep; 14(3): 196-202

14. Salwa Hagag Abdelaziz, Hala Ibrahim Abo Deif, Amel Dawod Goudia. Predictors of Breast Cancer Risk among Hair dye users at El-Manial University Hospital Cairo University, Egypt. MJN 2018 Vol 9 (3)

15. Yu-Tang Gao, Xiao-Ou Shu, Qi Dai, John D. Potter, Louise A Brinton, Wanqing Wen. Association of menstrual and reproductive factors with breast cancer risk: Results from Shanghai breast cancer study. Int J Cancer, June 2000; Vol 87

16. Farhana Badar, Shahid Mahmood, Raqib Faraz, Aneel Yousaf, Ain ul Quader, Hena Asif et al. Epidemiology of Breast Cancer at Shaukat Khanum Memorial Cancer Hospital \& Research Centre, Lahore. JCPSP 2015; Vol. 25 (10): 738-742

17. Gulshan Ali Memon, Roshan Ali Solangi, Amir Hamza Dahri, Atta Muhammad Chandio. An Experience with Breast Cancer at P.M.C.H Nawabshah. IJSR. Med Channel Oct-Dec 1999; 5 (4): 7-14

18. Mansha M, et al. Prevalence of known Risk Factors in Women, Diagnosed with breast cancer in Inmol Hospital, Lahore, Punjab. Asian Pac J Cancer Pre, 2016

19. T. Pervez, MS Anwar, Am Sheikh. Study of Risk Factors for Carcinoma Breast in Adult General population in Lahore. JCPSP 11 (5), 291-293, 2001

20. A Ahmed, F Naz, MA Khawaja, AU Haq, SN Malik, SA Khan. Presentation of Carcinoma Breast in Pakistan- A study of 60 Cases. Annals of KEMU. 2000; 6 (4)

21. Muhammad Faheem, Muhammad Khurram, Imran Ali Jafri, Humera Mahmood, Zubair Hussain, Ghulam Sabir Iqbal, et al. Risk Factors for Breast Cancer in Patients treated at NORI Hospital, Islamabad. J. Pak. Med. Assoc. 2007; 57(5): 242

22. Lina S. Morch, Ph. D., Charlotte W. Skovlund, M. Sc., Philip C. Hannaford, M.D., Lisa Iversen, Ph. D., et al. Contemporary Hormonal Contraception and the Risk of Breast Cancer. N Engl J Med 2017; 377:2228-2239

23. Memon ZA, et al. Clinical presentation and Frequency of Risk factors in Patients with Breast Carcinoma in Pakistan. Asian Pac J Cancer Prev. 2015 
24. Rukhsana khursheed, Mubasshir A. Sheikh and Farida Munnawar. Geographic/Physiological Diversity of Female Breast Cancer Among Developing and Developed Countries. PJMS, 2001; 17 (2): 99-105

25. Ibid

26. Llanos AAM, Rabkin A, Bandersa EV, Zirpoli G, Gozalez BD, Xing CY, Qin B. et al. Hair product use and breast cancer risk among African American and white women. J Carcinog. 2017 Sep 1:38(9): 883-892
27. Sanna Heikkinen, Janne Pitkaniemi, Tytti sarkeala, Nea Malila, Markku Koskenvuo. Does Hair Dye Use Increase the Risk of Breast Cancer? A Population-Based CaseControl Study of Finnish Women. PloS ONE; 10 (8): 2015

28. Ritika Gera, Ramia Mokbel, Ivanna Igor, Kefah Mokbel Does the Use of Hair Dyes Increase the Risk of Developing Breast Cancer? A meta-analysis \& review of literature. Anticancer research 2018; 38 (2): 707-717 